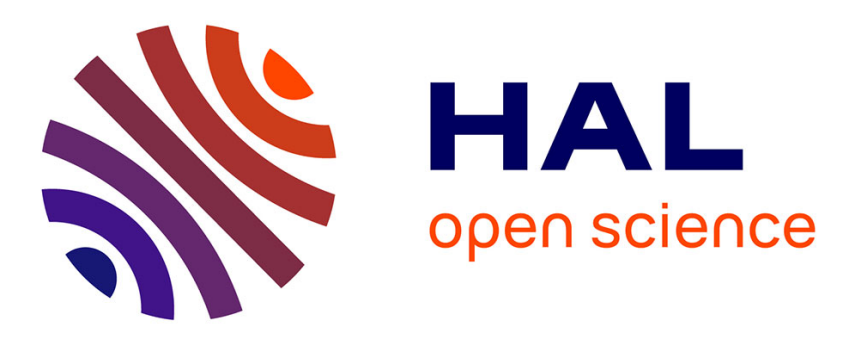

\title{
Visual Servoing of an Airplane for Alignment with respect to a Runway
}

O. Bourquardez, François Chaumette

\section{To cite this version:}

O. Bourquardez, François Chaumette. Visual Servoing of an Airplane for Alignment with respect to a Runway. IEEE Int. Conf. on Robotics and Automation, ICRA'07, 2007, Rome, Italy. pp.1330-1335. inria-00350635

\section{HAL Id: inria-00350635 https://hal.inria.fr/inria-00350635}

Submitted on 7 Jan 2009

HAL is a multi-disciplinary open access archive for the deposit and dissemination of scientific research documents, whether they are published or not. The documents may come from teaching and research institutions in France or abroad, or from public or private research centers.
L'archive ouverte pluridisciplinaire HAL, est destinée au dépôt et à la diffusion de documents scientifiques de niveau recherche, publiés ou non, émanant des établissements d'enseignement et de recherche français ou étrangers, des laboratoires publics ou privés. 


\title{
Visual Servoing of an Airplane for Alignment with respect to a Runway
}

\author{
Odile Bourquardez and François Chaumette
}

\begin{abstract}
In this paper, we propose a visual servoing scheme to align an airplane with respect to a runway. A linearized model of the airplane dynamics and decoupled visual features are designed to build the control scheme. Simulation results are obtained with a quite realistic flight simulator which is based on a non linear airplane dynamic model. They show that the airplane realizes correctly the specified task by using visual data.
\end{abstract}

\section{INTRODUCTION}

Visual servoing schemes enable to control robot motion by using visual features as feedback signal [2] [5]. The camera can be embedded on the robot or be placed in its external environment. Different kinds of visual features can be used to design the control law: 2D data, directly extracted from the image, or 3D data, provided by a preliminary pose estimation (a mixture of the both can also be used [7]).

Using visual servoing to control aircrafts yields new problems. Indeed, in most robotics applications, the degrees of freedom can be assumed to be as a pure integrator leading to kinematics control schemes. But designing visual servoing for aircrafts requires to take into account their dynamic constraints.

In a lot of works, camera is used to provide pose estimation. The control law is then based on the position, and other sensors such as GPS or inertial sensors can be used in the control law. This strategie is used in [8] [11] [12] to control helicopters. In this paper, we have chosen to not deal with pose estimation, but to consider $2 \mathrm{D}$ visual servoing. The most common 2D approach to control aircrafts is to consider state equations and to link the state vector with the visual features. For instance, in [14] the authors incorporate the dynamics of a blimp in the equations of the image dynamics, and apply a PID control. In [1] [9] [10] the state model is linearized around an equilibrium state and the visual features are used as output. Then standard control design such as LQR (Linear Quadratic Regulator) can be used with the LTI (Linear Time Invariant) system obtained. We have used a similar approach for the longitudinal control. However, the proposed lateral control is based on a simple constraint applied to the lateral position.

Several strategies have already been used to follow linear structures by visual servoing. In [6], the binormalized Plücker coordinates of the lines are used, and the dynamic properties of helicopters are exploited to design a control

O. Bourquardez is with IRISA-CNRS, Campus de Beaulieu, 35042 Rennes cedex, France odile.bourquardez@irisa.fr

F. Chaumette is with IRISA-INRIA, Campus de Beaulieu, 35042 Rennes cedex, France francois.chaumette@irisa.fr scheme based on a Lyapunov function and backstepping techniques. In [10] vanishing point and vanishing line have been considered in a 2D visual servoing scheme for controlling a blimp. The scheme presented in [13] uses only two lines, and the camera is thus combined with other sensors in order to fully control the state parameters of a blimp. Lateral position of a small autonomous aircraft with respect to a road is performed in [3] by partial 3D pose estimation. In our case, we have designed new decoupled visual features in order to improve the relationship with the aircraft state.

In this paper, we consider three parallel lines, which could belong to a road or a runway for example. The aim is to follow this structure with constant attitude, velocity, and altitude. An airplane equipped with a fly-by-wire system is considered: the pilot commands are converted to electronic signals, and flight control computers determine how best to move the actuators to provide the desired response.

The relationship between the pilot commands and the aircraft motion is given by a linearized model (Section II). Then new visual features with suitable properties are designed from the image lines parameters (Section III). They enable to link the aircraft motion with the image motion. Finally, the image features are related to the control inputs, and a visual control scheme is designed in Section IV. Simulation results are shown Section V. Instead of being performed with the linearized model, the simulations use a more complete and realistic flight simulator. They show that the proposed control scheme enables the aircraft to realize correctly the specified task.

\section{AIRCRAFT MODEL}

\section{A. Control Inputs}

In most of previous works, the control inputs are actuators deflection and thrust produced by the propellers. In this paper, the aircraft considered is equipped with a fly-bywire system: instead of using cables, computer-generated electrical signals are used to transmit the pilot commands to the flight control surfaces. The fly-by-wire system provides additionally safety control to prevent insecure pilot commands. For safety reasons, it is thus preferable to leave unchanged this low-level flight control system and to deal with the pilot commands. Thus the considered inputs to control the aircraft are the throttle position and the control stick.

Let $\mathbf{u}=\left(\delta_{m}, \delta_{l}, \delta_{T}\right)$ denote the input vector. $\delta_{m} \in[-1 ; 1]$ is the control stick forward-backward position, and it mainly controls the angle of attack. $\delta_{l} \in[-1 ; 1]$ is the control stick left-right position, and it mainly induces rotation around the 
longitudinal axis. Finally $\delta_{T} \in[0 ; 1]$ is the throttle position, and it is related to thrust level. However, due to the aircraft dynamics, these movements are coupled and induce other trajectory or attitude modifications.

\section{B. State Components and other Notations}

Let $\mathcal{R}_{a}$ denote the aircraft frame, and $\mathcal{R}_{f}$ a fixed reference frame (Fig. 1). The aircraft pose is defined by the translation and the rotation between $\mathcal{R}_{a}$ and $\mathcal{R}_{f}$. Let $(X, Y, Z)$ denote the three components of the translation, and $(\phi, \theta, \psi)$ denote the roll-pitch-yaw angles of the rotation. Let $\mathbf{P}=(X, Y, Z, \phi, \theta, \psi)$ denote the pose vector.

The primary way for a pilot to change the airplane direction is to change the aircraft attitude. Let $\mathbf{v}_{a}=$ $\left(v_{a_{x}}, v_{a_{y}}, v_{a_{z}}, \omega_{a_{x}}, \omega_{a_{y}}, \omega_{a_{z}}\right)$ denote the aircraft instantaneous velocity (see Fig. 1.a), and $\boldsymbol{v}_{a}=\left(v_{a_{x}}, v_{a_{y}}, v_{a_{z}}\right)$ its translational velocity, $v_{a}$ its module, and $\dot{v}_{a}$ the module of the acceleration. Note that in most cases the velocity vector $\boldsymbol{v}_{a}$ and the aircraft longitudinal axis $x_{a}$ have not the same direction. Let $\alpha$ denote the angle of attack, between these two directions (see Fig. 1.b).

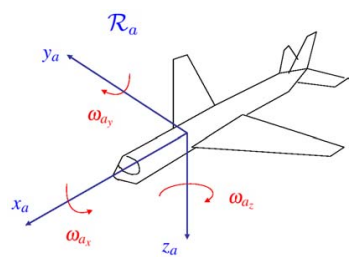

(a)

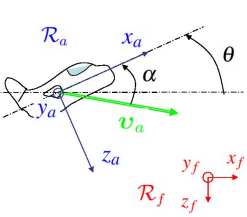

(b)
Fig. 1. (a) Aircraft frame and associated rotations, (b) Angles definitions

\section{Linearized Model}

The airplane dynamics model which links the pilot inputs and the aircraft motions have been provided by the French company Dassault Aviation. We have first linearized this model to design the control scheme, but the real one has been used to validate the control scheme. For that, the classical hypothesis of small perturbations around a trim flight is considered. Since the objective is to align the aircraft with the runway axis, the rotation matrix ${ }^{f} \mathbf{R}_{a}$ between the fixed frame and the aircraft frame is assumed to be close to identity, which allows the following approximation to be done:

$$
\dot{\mathbf{P}}=\mathbf{v}_{a} \text {. }
$$

Finally the aircraft model can be represented by the following state space equation: $\dot{\mathbf{x}}=\mathbf{A x}+\mathbf{B u}$ with $\mathbf{x}=$ $\left(\dot{\phi}, \dot{\psi}, \psi, Y, \alpha, \dot{\theta}, \theta, v_{a}, \dot{v}_{a}, Z\right)$. This model can be decoupled between the lateral motion in the horizontal plane (2), and the longitudinal motion in the vertical plane (3), where $\tilde{w}=$ $w-w^{*}$ represents the variation between the current value $w$ and the equilibrium value $w^{*}$ for any $w$ parameter. The constants $a_{1}, \ldots, a_{21}, b_{1}, \ldots, b_{4}$ are determined from aircraft constants and state equilibrium values.

$$
\left[\begin{array}{c}
\tilde{\ddot{\phi}} \\
\tilde{\ddot{\psi}} \\
\tilde{\dot{\psi}} \\
\tilde{\dot{Y}}
\end{array}\right]=\left[\begin{array}{cccc}
a_{1} & a_{2} & 0 & 0 \\
a_{3} & a_{4} & 0 & 0 \\
0 & 1 & 0 & 0 \\
0 & 0 & a_{5} & 0
\end{array}\right]\left[\begin{array}{c}
\tilde{\dot{\phi}} \\
\tilde{\psi} \\
\tilde{\psi} \\
\tilde{Y}
\end{array}\right]+\left[\begin{array}{c}
b_{1} \\
b_{2} \\
0 \\
0
\end{array}\right] \tilde{\delta}_{l}
$$

$$
\begin{aligned}
{\left[\begin{array}{c}
\tilde{\dot{\alpha}} \\
\tilde{\ddot{\theta}} \\
\tilde{\dot{\theta}} \\
\tilde{\dot{v}}_{a} \\
\tilde{\ddot{v}}_{a} \\
\tilde{\dot{Z}}
\end{array}\right]=} & {\left[\begin{array}{cccccc}
a_{6} & a_{7} & a_{8} & a_{9} & a_{10} & 0 \\
a_{11} & a_{12} & 0 & a_{13} & 0 & 0 \\
0 & 1 & 0 & 0 & 0 & 0 \\
0 & 0 & 0 & 0 & 1 & 0 \\
a_{14} & a_{15} & a_{16} & a_{17} & a_{18} & 0 \\
a_{19} & 0 & a_{20} & a_{21} & 0 & 0
\end{array}\right]\left[\begin{array}{c}
\tilde{\alpha} \\
\tilde{\dot{\theta}} \\
\tilde{\theta} \\
\tilde{v}_{a} \\
\tilde{\dot{v}}_{a} \\
\tilde{Z}
\end{array}\right] } \\
& +\left[\begin{array}{cc}
0 & 0 \\
b_{3} & 0 \\
0 & 0 \\
0 & 0 \\
0 & b_{4} \\
0 & 0
\end{array}\right]\left[\begin{array}{c}
\tilde{\delta}_{m} \\
\tilde{\delta}_{T}
\end{array}\right]
\end{aligned}
$$

\section{VISUAL FEATURES}

Visual features based on the measurements that can be extracted from the image of a runway are considered: they are its two border lines and its central line. These three lines are parallel in the 3D space, but due to the perspective projection model they intersect in the image plane at the vanishing point.

Since the objective is to align the aircraft with the runway axis, the desired camera pose is chosen as shown on Fig. 2: the optical axis is aligned with the runway axis and the image plane is orthogonal to the runway plane. The camera frame and the frame of reference $\mathcal{R}_{f}$ are depicted on Fig. 2. Let $H$ denote the camera altitude and $L$ the runway width. $\mathcal{L}_{l}$ is the left border line, $\mathcal{L}_{r}$ the right one, and $\mathcal{L}_{c}$ the central one.

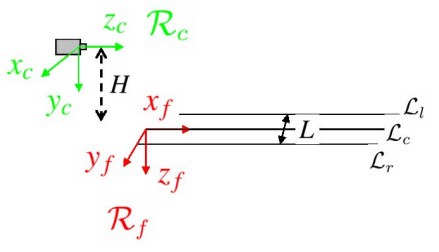

Fig. 2. Desired camera position

Let $s$ denote a visual feature. The relationship between $s$ and the camera instantaneous velocity $\mathbf{v}_{\mathbf{c}}=$ $\left(v_{c_{x}}, v_{c_{y}}, v_{c_{z}}, \omega_{c_{x}}, \omega_{c_{y}}, \omega_{c_{z}}\right)$ is defined by the well-known interaction matrix $\mathbf{L}_{s}$ [2] such that:

$$
\dot{s}=\mathbf{L}_{s} \mathbf{v}_{\mathbf{c}}
$$

\section{A. Straight Lines}

Each runway line projects as a straight line in the image plane, which is represented by the $(\rho, \theta)$ parameters such that $x \cos \theta+y \sin \theta-\rho=0$ (see Fig. 3).

A 3D line can be defined in the camera frame as the intersection of two planes:

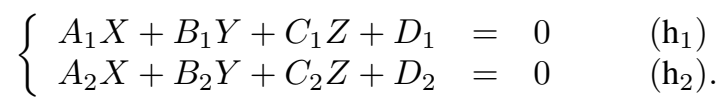

Then the corresponding interaction matrix is given by [2]:

$$
\mathbf{L}_{\theta}=\left[\begin{array}{llllll}
\lambda \cos \theta & \lambda \sin \theta & -\lambda \rho & -\rho \cos \theta & -\rho \sin \theta & -1
\end{array}\right],
$$

where $\lambda=\left(A_{i} \sin \theta-B_{i} \cos \theta\right) / D_{i}$. If we exclude the degenerated case where the projection center belongs to the straight line $\left(D_{1}=D_{2}=0\right)$, we can choose $D_{i} \neq 0$. 


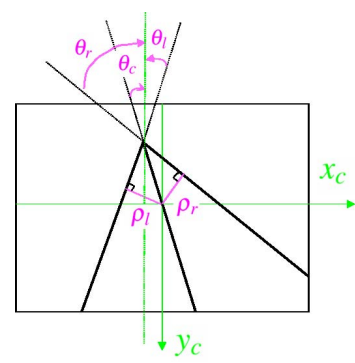

Fig. 3. $(\rho, \theta)$ parameters

If the lines are defined by using the horizontal plane (equation $Y-H=0$ in the desired camera frame, thus $(A, B, C, D)=(0,1,0,-H))$, the interaction matrix can be written as:

$$
\begin{aligned}
& \mathbf{L}_{\theta_{l}}=\left[\begin{array}{llllll}
\frac{1}{H} \mathrm{c}^{2} \theta_{l} & \frac{1}{H} \mathrm{c} \theta_{l} \mathrm{~s} \theta_{l} & -\frac{1}{H} \rho_{l} \mathrm{c} \theta_{l} & -\rho_{l} \mathrm{c} \theta_{l} & -\rho_{l} \mathrm{~s} \theta_{l} & -1
\end{array}\right] \\
& \mathbf{L}_{\theta_{r}}=\left[\begin{array}{llllll}
\frac{1}{H} \mathrm{c}^{2} \theta_{r} & \frac{1}{H} \mathrm{c} \theta_{r} \mathrm{~s} \theta_{r} & -\frac{1}{H} \rho_{r} \mathrm{c} \theta_{r} & -\rho_{r} \mathrm{c} \theta_{r} & -\rho_{r} \mathrm{~s} \theta_{r} & -1
\end{array}\right] \\
& \mathbf{L}_{\theta_{c}}=\left[\begin{array}{llllll}
\frac{1}{H} \mathrm{c}^{2} \theta_{c} & \frac{1}{H} \mathrm{c} \theta_{c} \mathrm{~s} \theta_{c} & -\frac{1}{H} \rho_{c} \mathrm{c} \theta_{c} & -\rho_{c} \mathrm{c} \theta_{c} & -\rho_{c} \mathrm{~s} \theta_{c} & -1
\end{array}\right]
\end{aligned}
$$

where $\mathrm{c} \theta=\cos \theta, \mathrm{s} \theta=\sin \theta$, and $\mathrm{c}^{2} \theta=(\cos \theta)^{2}$.

Instead of using the horizontal plane, we can choose the vertical planes: equation $X+L / 2=0$ for $\mathcal{L}_{l}$, and $X-L / 2=$ 0 for $\mathcal{L}_{r}$ in the desired camera frame. (Note that the vertical plane can not be used for the central line, because the optical center belongs to the plane $X=0$, and thus $D=0$ ). This choice enables to obtain the parameter $L$ instead of $H$ in the interaction matrix. It is interesting in practice since $H$ is variable and has to be estimated, on the contrary to $L$ which is constant and can be assumed to be known. The interaction matrix can be written as

$$
\begin{aligned}
\mathbf{L}_{\theta_{l}} & =\left[\begin{array}{rrrrrr}
\frac{2}{L} \mathrm{~s} \theta_{l} \mathrm{c} \theta_{l} & \frac{2}{L} \mathrm{~s}^{2} \theta_{l} & -\frac{2}{L} \rho_{l} \mathrm{~s} \theta_{l} & -\rho_{l} \mathrm{c} \theta_{l} & -\rho_{l} \mathrm{~s} \theta_{l} & -1
\end{array}\right] \\
\mathbf{L}_{\theta_{r}} & =\left[\begin{array}{llllll}
-\frac{2}{L} \mathrm{~s} \theta_{r} \mathrm{c} \theta_{r} & -\frac{2}{L} \mathrm{~s}^{2} \theta_{r} & \frac{2}{L} \rho_{r} \mathrm{~s} \theta_{r} & -\rho_{r} \mathrm{c} \theta_{r} & -\rho_{r} \mathrm{~s} \theta_{r} & -1
\end{array}\right] .
\end{aligned}
$$

Note that, in the desired position of the camera, $H$ and $L$ are related by $\theta_{l}=\arctan \left(\frac{L}{2 H}\right)$ and $\theta_{r}=-\arctan \left(\frac{L}{2 H}\right)$ which gives

$$
H=\frac{L}{2 \tan \left(\frac{\theta_{l}-\theta_{r}}{2}\right)} .
$$

The desired values of the lines parameters are (see Fig. 2): $\rho_{c}^{*}=\rho_{l}^{*}=\rho_{r}^{*}=0, \theta_{c}^{*}=0$, and $\theta_{l}^{*}=-\theta_{r}^{*}=\arctan \left(\frac{L}{2 H}\right)$.

In order to obtain interaction matrices with good decoupling properties, new visual features are defined from $\theta_{r}, \theta_{l}$ and $\theta_{c}$ :

$$
\begin{aligned}
\delta & =\theta_{l}+\theta_{r}-2 \theta_{c}, \\
\sigma & =\theta_{l}-\theta_{r} .
\end{aligned}
$$

Recalling (7) and using the desired values of the lines parameters, we obtain only one non-zero term in the interaction matrix $\mathbf{L}_{\delta}$ computed for the desired airplane pose:

$$
\mathbf{L}_{\delta}^{*}=\left[\frac{1}{H}\left(\mathrm{c}^{2} \theta_{l}+\mathrm{c}^{2} \theta_{r}-2\right) \quad 0 \quad 00 \quad 0 \quad 000\right]
$$

where the altitude $H$ is either estimated using (9) or given by other sensors.

Then, recalling (8) and using the desired values of the lines parameters, we obtain also only one non-zero term in the desired interaction matrix $\mathbf{L}_{\sigma}^{*}$ :

$$
\mathbf{L}_{\sigma}^{*}=\left[\begin{array}{lllllll}
0 & \frac{2}{L}\left(\mathrm{~s}^{2} \theta_{l}+\mathrm{s}^{2} \theta_{r}\right) & 0 & 0 & 0 & 0
\end{array}\right] .
$$

\section{B. Vanishing Point}

The vanishing point is well known to be invariant with respect to any 3D translational motion. This property is particularly interesting to design visual features able to control the rotational motions.

The relationship between the camera velocity and the vanishing point coordinates $\left(x_{f}, y_{f}\right)$ is given by the interaction matrix $\mathbf{L}_{x_{f}}$ and $\mathbf{L}_{y_{f}}[10]$ :

$$
\begin{aligned}
\mathbf{L}_{x_{f}} & =\left[\begin{array}{lllccr}
0 & 0 & 0 & x_{f} y_{f} & -\left(1+x_{f}^{2}\right) & y_{f}
\end{array}\right] \\
\mathbf{L}_{y_{f}} & =\left[\begin{array}{lllccr}
0 & 0 & 0 & \left(1+y_{f}^{2}\right) & -x_{f} y_{f} & -x_{f}
\end{array}\right] .
\end{aligned}
$$

The desired values of the vanishing point coordinates are $\left(x_{f}^{*}, y_{f}^{*}\right)=(0,0)$ (see Fig. 2). The desired interaction matrix can then be written as:

$$
\begin{aligned}
& \mathbf{L}_{x_{f}}^{*}=\left[\begin{array}{llllrl}
0 & 0 & 0 & 0 & -1 & 0
\end{array}\right] \\
& \mathbf{L}_{y_{f}}^{*}=\left[\begin{array}{llllrl}
0 & 0 & 0 & 1 & 0 & 0
\end{array}\right] .
\end{aligned}
$$

\section{VISUAL SERVOING}

We now link the airplane dynamic model, presented in Section II, and the visual features model, presented in the last section, to design a visual servoing control scheme.

\section{A. From Camera Velocity to Aircraft Velocity}

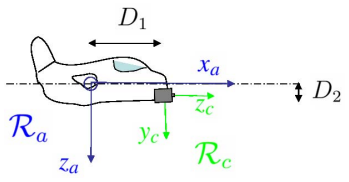

Fig. 4. Camera position on the aircraft

The camera is fixed on the front of the aircraft, as can be seen on Fig. 4. Let ${ }^{c} \mathbf{R}_{a}$ and ${ }^{c} \mathbf{t}_{a}$ denote respectively the rotation matrix and the translation vector between aircraft frame and camera frame.

The link between the camera velocity screw $\left(\mathbf{v}_{c}\right)$ and the aircraft velocity screw $\left(\mathbf{v}_{a}\right)$ is given by [2]:

$$
\mathbf{v}_{c}={ }^{c} \mathbf{S}_{a} \mathbf{v}_{a}
$$

with ${ }^{c} \mathbf{S}_{a}=\left[\begin{array}{cc}{ }^{c} \mathbf{R}_{a} & {\left[{ }^{c} \mathbf{t}_{a}\right] \times{ }^{c} \mathbf{R}_{a}} \\ \mathbf{0}_{3 \times 3} & { }^{c} \mathbf{R}_{a}\end{array}\right]$. Note that $\mathbf{0}_{3 \times 3}$ is the null square matrix of order 3 , and $\left[{ }^{c} \mathbf{t}_{a}\right]_{\times}$is the skew symmetric matrix such that $\left[{ }^{c} \mathbf{t}_{a}\right]_{\times} \mathbf{w}={ }^{c} \mathbf{t}_{a} \times \mathbf{w}$ for any vector $\mathbf{w}$.

In our case, we have:

$$
{ }^{c} \mathbf{R}_{a}=\left[\begin{array}{lll}
0 & 1 & 0 \\
0 & 0 & 1 \\
1 & 0 & 0
\end{array}\right] \quad ; \quad{ }^{c} \mathbf{t}_{a}=\left[\begin{array}{c}
0 \\
-D_{2} \\
-D_{1}
\end{array}\right]
$$

In practice, $D_{1}$ and $D_{2}$ are a few meters. The corresponding terms will act during the transient as small perturbations: neglecting them does not imply significantly different results.

We thus consider:

$$
{ }^{c} \mathbf{S}_{a}=\left[\begin{array}{cc}
{ }^{c} \mathbf{R}_{a} & \mathbf{0}_{3 \times 3} \\
\mathbf{0}_{3 \times 3} & { }^{c} \mathbf{R}_{a}
\end{array}\right] .
$$




\section{B. Links between Visual Features and Aircraft State}

Using the desired interaction matrix is a classical approximation in visual servoing when the considered displacements are small. Using this approximation, and recalling (4) and (16) gives:

$$
\dot{s}=\mathbf{L}_{s}^{* c} \mathbf{S}_{a} \mathbf{v}_{a} .
$$

We consider the equilibrium position. Since equilibrium rotation between $\mathcal{R}_{a}$ and $\mathcal{R}_{f}$ is assumed to be close to the identity, recalling (1) gives:

$$
\dot{s}=\mathbf{L}_{s}^{* c} \mathbf{S}_{a} \dot{\mathbf{P}} .
$$

Since $\mathbf{L}_{s}^{*}$ and ${ }^{c} \mathbf{S}_{a}$ are constant, the above equation can then be integrated, for small displacements around the equilibrium position [1], [4]. We obtain:

$$
\tilde{s}=\mathbf{L}_{s}^{* c} \mathbf{S}_{a} \tilde{\mathbf{P}}
$$

where the $\sim$ notation represents the variation between the current value and the equilibrium value, as introduced in Section II-C.

Using the visual features $\delta, \sigma, x_{f}, y_{f}$ in (20), and recalling (12), (13), (15) and (17) gives:

$$
\begin{aligned}
\tilde{\delta} & =\frac{1}{H}\left(\mathrm{c}^{2} \theta_{l}+\mathrm{c}^{2} \theta_{r}-2\right) \tilde{Y} \\
\tilde{\sigma} & =\frac{2}{L}\left(\mathrm{~s}^{2} \theta_{l}+\mathrm{s}^{2} \theta_{r}\right) \tilde{Z} \\
\tilde{x}_{f} & =-\tilde{\psi} \\
\tilde{y}_{f} & =\tilde{\theta},
\end{aligned}
$$

from which we deduce directly:

$$
\begin{aligned}
& \tilde{\dot{x}}_{f}=-\tilde{\dot{\psi}} \\
& \tilde{\dot{y}}_{f}=\tilde{\dot{\theta}} .
\end{aligned}
$$

Note that two singular cases can theoretically be encountered with (21):

- $H \rightarrow 0$ : This is not a problem since in practice the camera altitude is never null.

- $\left(\theta_{l}, \theta_{r}\right) \rightarrow\left(k_{1} \pi, k_{2} \pi\right)$ : this case would occur when the optical axis is vertical, or if the altitude $H$ is infinite. In practice this is not a problem since such configurations are impossible with the aircraft.

Finally, note that the singularity that can be encountered with (22) is the same as the previous one.

The relative lateral position $\tilde{Y}$ and altitude $\tilde{Z}$ can be linked with visual features by inverting the equations (21) and (22):

$$
\begin{aligned}
\tilde{Y} & =\frac{H}{\mathrm{c}^{2} \theta_{l}+\mathrm{c}^{2} \theta_{r}-2} \tilde{\delta}, \\
\tilde{Z} & =\frac{L}{2\left(\mathrm{~s}^{2} \theta_{l}+\mathrm{s}^{2} \theta_{r}\right)} \tilde{\sigma} .
\end{aligned}
$$

By using the last line of (3), $\tilde{\alpha}$ can be expressed as a linear function of $\tilde{\dot{Z}}, \tilde{\theta}$, and $\tilde{v}_{a}$. Assuming that $\mathrm{s}^{2} \theta_{l}+\mathrm{s}^{2} \theta_{r}$ is constant and deriving (28) gives a link between $\tilde{\dot{Z}}$ and $\tilde{\dot{\sigma}}$. The link between $\tilde{\theta}$ and $\tilde{y}_{f}$ is given by (24). Finally, we obtain:

$$
\tilde{\alpha}=\frac{L}{2 a_{19}\left(\mathrm{~s}^{2} \theta_{l}+\mathrm{s}^{2} \theta_{r}\right)} \tilde{\dot{\sigma}}-\frac{a_{20}}{a_{19}} \tilde{y}_{f}-\frac{a_{21}}{a_{19}} \tilde{v}_{a} .
$$

$\tilde{\boldsymbol{v}}_{a}$ can not be entirely determined by using the three parallel lines. Other visual feature should be used in order to determine the longitudinal component of the velocity, along the runway axis. Since none is easily available, $\tilde{v}_{a}$ is assumed to be known by mean of other sensors than the camera.

\section{Control Law}

1) Lateral Control: The horizontal motions are controlled by the lateral control: when the equilibrium flight is modified by small perturbations, the control stick left-right position allows to drive the aircraft to the desired equilibrium roll, heading, and lateral position. It is intuitive to see that, in the absence of crosswind, if the lateral position $Y$ is stabilized to 0 , the heading $\psi$ is stabilized to 0 , and that implies that the airplane roll $\phi$ is null too. Thus the idea to design the control law is to link the control input $\delta_{l}$ with the lateral position $Y$, in order to drive it to 0 .

It is well-known that the right-left position of the control stick enables to control the roll rate. Thus we can consider a linear link between $\delta_{l}$ and $\dot{\phi}: \dot{\phi}=\lambda \tilde{\delta}_{l}$, with $\lambda>0$.

- Remark: Note that using the first line of (2) enables to retry this relationship, and to determine the $\lambda$ coefficient. Assuming that $\delta_{l}$ is constant and integrating the differential equation $\tilde{\ddot{\phi}}(t)=a_{1} \tilde{\dot{\phi}}(t)+b_{1} \tilde{\delta}_{l}$ gives

$$
\tilde{\dot{\phi}}=c_{1} e^{a_{1} t}-\frac{b_{1}}{a_{1}} \tilde{\delta}_{l}
$$

with $c_{1}$ constant, and $a_{1}<0, b_{1}>0$. Since $a_{1}<0$, the exponential term can be neglected, and we obtain the following linear relationship:

$$
\tilde{\dot{\phi}}=-\frac{b_{1}}{a_{1}} \tilde{\delta}_{l} \text {. }
$$

Using the second line of (2), and the fact that $a_{4}$ and $b_{2}$ are negligible, we obtain a direct relationship between $\ddot{\psi}$ and $\dot{\phi}$ :

$$
\tilde{\ddot{\psi}}=a_{3} \tilde{\dot{\phi}}, \text { with } \quad a_{3}>0 .
$$

Then using (32) in (31) gives:

$$
\tilde{\delta}_{l}=-\frac{a_{1}}{b_{1} a_{3}} \tilde{\ddot{\psi}}
$$

Using the last line of (2) enables to relate $\psi$ and $\dot{Y}$ :

$$
\tilde{\dot{Y}}=a_{5} \tilde{\psi} \text {, with } a_{5}>0 .
$$

Taking its time derivative, we obtain

$$
\tilde{\ddot{Y}}=a_{5} \tilde{\dot{\psi}} \text {. }
$$

Then deriving again yields $\tilde{\ddot{Y}}=a_{5} \tilde{\ddot{\psi}}$, and recalling (33) gives

$$
\tilde{\delta}_{l}=-\frac{a_{1}}{b_{1} a_{3} a_{5}} \tilde{\ddot{Y}} .
$$


The following constraint is chosen: $\tilde{Y}=-k_{1} \tilde{\ddot{Y}}-k_{2} \tilde{\dot{Y}}-k_{3} \tilde{Y}$, where $k_{1}, k_{2}, k_{3}$ are chosen such that $\tilde{Y}$ is stable. Replacing $\tilde{\ddot{Y}}$ in (36), the lateral control law can finally be written as:

$$
\tilde{\delta}_{l}=-n_{1} \tilde{\ddot{Y}}-n_{2} \tilde{\dot{Y}}-n_{3} \tilde{Y}
$$

where $n_{i}=\left(-k_{i} a_{1}\right) /\left(b_{1} a_{3} a_{5}\right)$.

Then the parameters can be substituted by their visual equivalents: using (23) and (25) in (34) and (35) gives

$$
\begin{aligned}
\tilde{\dot{Y}} & =-a_{5} \tilde{x}_{f} \\
\tilde{\ddot{Y}} & =-a_{5} \tilde{\dot{x}}_{f} .
\end{aligned}
$$

Substituting (27), (38), and (39) in (37) gives the visual control law:

$$
\tilde{\delta}_{l}=n_{1} a_{5} \tilde{\dot{x}}_{f}+n_{2} a_{5} \tilde{x}_{f}-n_{3} \frac{H}{\mathrm{c}^{2} \theta_{l}+\mathrm{c}^{2} \theta_{r}-2} \tilde{\delta} .
$$

We recall that the altitude $H$ can be estimated using (9), or measured by other sensors. As for parameter $\dot{x}_{f}$, it can be obtained from the image using $\dot{x}_{f}(k)=\frac{x_{f}(k)-x_{f}(k-1)}{\Delta t}$, or directly measured by a gyroscope, since it is related to the yaw rate (see (25)). All other parameters can be directly determined from the image.

2) Longitudinal Control: The vertical motions are controlled by the longitudinal control: when the equilibrium flight is modified by small perturbations, the throttle and the control stick forward-backward position allow to drive the aircraft to the desired equilibrium altitude, velocity, pitch and angle of attack.

Lateral control design is based on simple and intuitive links between input, roll rate, heading angle, and lateral position. Longitudinal state-space model (see (3)) shows coupling between the two concerned inputs, and at the view of this model, it is not easy to design simple control laws. Since the longitudinal state-space model is linear time invariant, the standard LQR optimal control can be used to design the longitudinal control law. The LQR regulator ensures the minimization of a quadratic cost function which takes into account the evolution of the state and the energy consumption. A state feedback matrix $\mathbf{K}$ can be computed in order to drive the system from an initial position to the trim position. With standard LQR regulator, the control law would be:

$$
\tilde{\mathbf{u}}_{l o}=-\mathbf{K} \tilde{\mathbf{x}}_{l o}
$$

where $\tilde{\mathbf{x}}_{l o}$ and $\tilde{\mathbf{u}}_{l o}$ are parts of the state vector and the input vector related to the longitudinal motion: $\tilde{\mathbf{x}}_{l o}=\left(\tilde{\alpha}, \tilde{\dot{\theta}}, \tilde{\theta}, \tilde{v}_{a}, \tilde{\dot{v}}_{a}, \tilde{Z}\right)$, and $\tilde{\mathbf{u}}_{l o}=\left(\tilde{\delta}_{m}, \tilde{\delta}_{T}\right)$.

Since the state vector is not directly measured, we use the visual features presented before and their links with the state $\tilde{\mathbf{x}}_{l o}$. Thus, recalling (29), (26), (24), and (28) the control law becomes (see Fig. 5):

$$
\tilde{\mathbf{u}}_{l o}=-\mathbf{K} \tilde{\mathbf{s}}
$$

with $\tilde{\mathbf{s}}=\left[\frac{L}{2 a_{19}\left(\mathrm{~s}^{2} \theta_{l}+\mathrm{s}^{2} \theta_{r}\right)} \tilde{\dot{\sigma}}-\frac{a_{20}}{a_{19}} \tilde{y}_{f}-\frac{a_{21}}{a_{19}} \tilde{v}_{a}, \tilde{\dot{y}}_{f}, \tilde{y}_{f}, \tilde{v}_{a}\right.$, $\left.\tilde{\dot{v}}_{a}, \frac{L}{2\left(\mathrm{~s}^{2} \theta_{l}+\mathrm{s}^{2} \theta_{r}\right)} \tilde{\sigma}\right]$.
As already explained, the velocity $v_{a}$ and the acceleration $\dot{v}_{a}$ are both assumed to be measured by other proprioceptive sensors. All other parameters can be determined from the image. The derived parameters are obtained by differentiating the corresponding values.

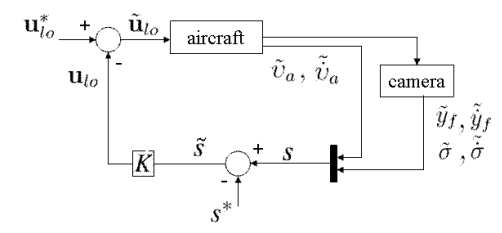

Fig. 5. Bloc diagram for longitudinal visual control

\section{RESULTS}

\section{A. Experimental Conditions}

The proposed control scheme has been tested in a simulation software based on a library provided by the French company Dassault Aviation. This library allows to simulate the behaviour of an aircraft equipped with a fly-by-wire control system. It can be controlled by the throttle and the control stick, and it provides pose and state measures.

In order to visualize the world where the aircraft is flying around, a visualizator is linked to the library. It allows to put a camera in the 3D environment (for example on the aircraft), and visualize the corresponding images (Fig. 6).

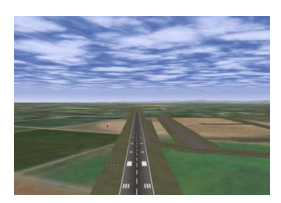

(a)

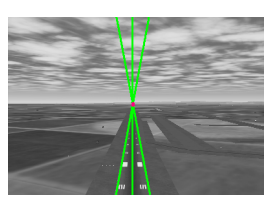

(b)
Fig. 6. (a) Runway image from embedded camera. (b) Lines and vanishing point.

The linearized model of the aircraft is the one presented in Section II-C. It has been used with the Control System Toolbox and the Matlab/Simulink tools to design the feedback matrix $\mathbf{K}$.

In order to test the presented control scheme, an equilibrium flight is considered. Perturbations are applied to drive the aircraft to a close state and position. Then the control laws (40) and (42) are used together to drive again the aircraft to its equilibrium flight.

\section{B. Results}

The aircraft lateral position is initially $Y \simeq 8.7 \mathrm{~m}$ whereas the runway takes place at $Y^{*}=0 \mathrm{~m}$, and the initial heading $\psi=-3 \mathrm{deg}$ whereas the runway heading is $\psi^{*}=0 \mathrm{deg}$. Before using the control laws, the equilibrium flight is submitted to perturbations with inappropriate inputs on the throttle and on the forward-backward control stick position. Consequently the altitude, velocity, angle of attack and pitch angle are driven close to their equilibrium state $\left(Z^{*}=-827\right.$ $\mathrm{m}, v_{a}^{*}=83 \mathrm{~m} / \mathrm{s}, \alpha^{*}=0.5 \mathrm{deg}$, and $\theta^{*}=0.5 \mathrm{deg}$ ).

In order to change its heading, the aircraft has first to turn around its longitudinal axis, to acquire roll $(\phi \neq 0)$. Then the aircraft turns: the heading $\psi$ is modified and consequently 
the lateral position $Y$ too (see Fig. 7). At the end of the control, the lateral position has been driven to $Y=Y^{*}=0$. Since the lateral position $Y$ is stabilized, the heading is null $\left(\psi=\psi^{*}=0\right)$. Since the heading is stabilized, the roll is null $\left(\phi=\phi^{*}=0\right)$. This motion is controlled by the right-left position of the control stick $\tilde{\delta}_{l}$ (see Fig.9.a), using the control law (40) and the following gains: $n_{1}=4.3, n_{2}=0.89, n_{3}=$ 0.045 . Note that the $x_{f}$ coordinate of the vanishing point has a symmetric behaviour as the heading $\psi$ (see Fig. 7.b, 10.a, and recall (23)).

The control law (42) enables to drive the longitudinal parameters to there equilibrium values, ie to drive the corresponding tilde values to zero (see Fig. 7.a, 8.a, 8.b). The throttle input and the backward-forward position of the control stick are used to realize correctly this task, and at the end they reach there equilibrium values too (see Fig. 9.b).

As can be seen on Fig. 10.a, the coordinates of the vanishing points reach their desired values. Figure 10.b shows that the orientation of the lines are finally stabilized. Note that they are such as $\theta_{c}^{*}=0$, and $\theta_{l}^{*}=-\theta_{r}^{*}$, as expected.

The proposed control scheme thus enables the aircraft to correct effects of perturbations from a close state, by using visual data.

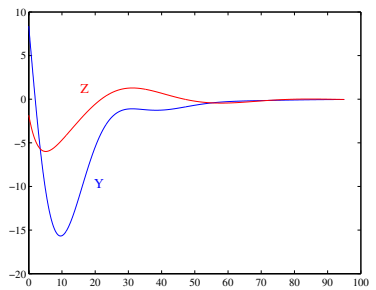

(a)

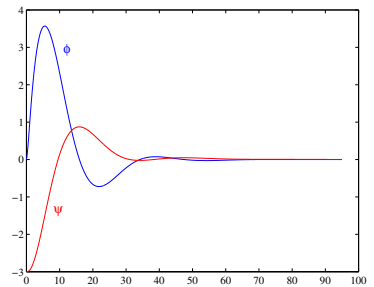

(b)
Fig. 7. Time evolution of the position parameters $\tilde{Y}$ and $\tilde{Z}$ (in meters) (a), and of the orientation angles $\tilde{\phi}$ and $\tilde{\psi}$ (in degrees) (b).

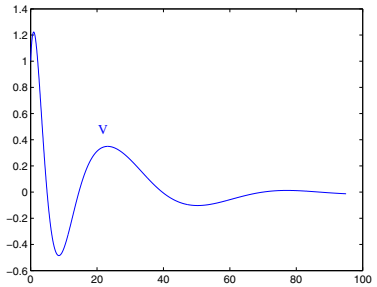

(a)

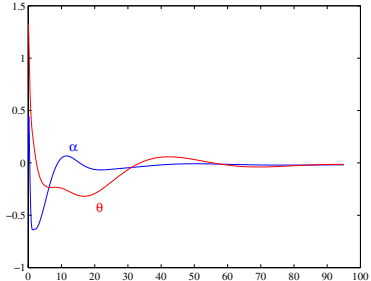

(b)
Fig. 8. Time evolution of the velocity $\tilde{v}_{a}$ (in meters per seconds) (a), and of the angles $\tilde{\alpha}$ and $\tilde{\theta}$ (in degrees) (b).

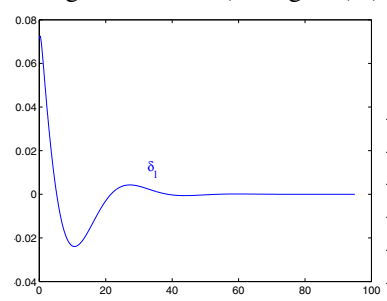

(a)

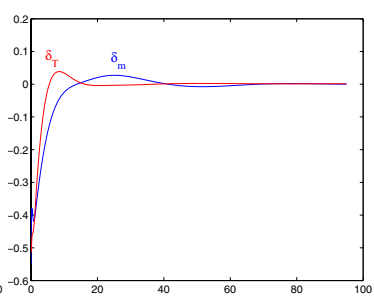

(b)
Fig. 9. Time evolution of the inputs $\tilde{\delta}_{l}, \tilde{\delta}_{T}$, and $\tilde{\delta}_{m}$

\section{CONCLUSION}

In this paper, a visual servoing scheme to align an aircraft with respect to a runway has been proposed. It uses a

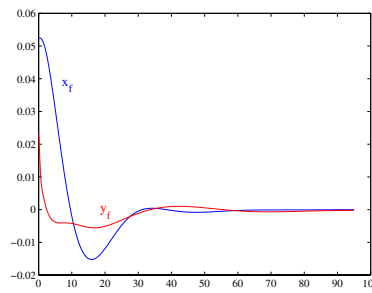

(a)

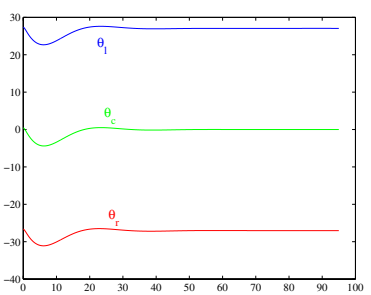

(b)
Fig. 10. Time evolution of the visual parameters: coordinates of the vanishing point $\left(\tilde{x}_{f}, \tilde{y}_{f}\right)$ (in meters) (a), and lines orientation $\theta_{l}, \theta_{r}, \theta_{c}$ (in degrees) (b).

linearized model of the airplane dynamics, and decoupled visual features. Simulation results show that the proposed control laws enable the airplane to be aligned with respect to the runway when its initial state and pose are close to the desired ones. Future work will be devoted to the application of alignment for automatic take-off and landing.

\section{ACKNOWLEDGMENTS}

This work has been supported by a DGA grant. The authors strongly thank Bruno Patin, Jean-Pierre Belmont, JeanPierre Tomaszower, Guillaume Rimbault and Erica Palmyre from Dassault Aviation for providing the flight simulator, the dynamical airplane model and for useful discussions.

\section{REFERENCES}

[1] J. R. Azinheira, P. Rives, J. R. H. Carvalho, G. F. Silveira, E. C. de Paiva, and S. S. Bueno, Visual Servo Control for the Hovering of an Outdoor Robotic Airship. ICRA'02, pp. 2787-2792, Vol. 3, Washington, USA, May 2002.

[2] B. Espiau, F. Chaumette, and P. Rives, A new approach to visual servoing in robotics, ITRA'92, 8(3):313-326, 1992.

[3] E. Frew, T. McGee, Z. Kim, X. Xiao, S. Jackson, M. Morimoto, S. Rathinam, J. Padial, R. Sengupta, Vision-Based Road Following Using a Small Autonomous Aircraft, IAC'04, Big Sky, MT, March 2004.

[4] J. Gangloff, M. de Mathelin, High speed visual servoing of a 6 DOF manipulator using MIMO predictive control, ICRA'00, pp. 3751-3756, Vol.4, San Francisco, CA, USA, April 2000.

[5] S. Hutchinson, G. Hager, P. I. Corke, A tutorial on visual servo control, ITRA'96, Vol.12, No. 5, pp. 651-670, 1996.

[6] R. Mahony, T. Hamel, Image-based visual servo control of aerial robotic systems using linear image features, ITRA'05, pp. 227- 239, Vol.21, April 2005.

[7] E. Malis, F. Chaumette, S. Boudet, 2 1/2 D visual servoing, ITRA'99, Vol.15(2), pp. 238-250, April 1999.

[8] L. Mejias, S. Saripalli, G. Sukhatme, P. Campoy, Detection and tracking of external features in a urban environment using an autonomous helicopter, ICRA'05, pp. 3983-3988, Barcelona, Spain, April 2005.

[9] P. Rives, J. R. Azinheira, Visual Auto-landing of an Autonomous Aircraft, Research Report INRIA-Sophia Antipolis, No 4606, November 2002.

[10] P. Rives, J. R. Azinheira, Linear Structures Following by an Airship using Vanishing Point and Horizon Line in Visual Servoing Scheme, ICRA'04, pp. 255-260, Vol.1, New Orleans, April 2004.

[11] S. Saripalli, J. F. Montgomery, G. S. Sukhatme, Visually-guided landing of an unmanned aerial vehicle, ITRA'03, Vol. 19, No. 3, pp. 371-381, 2003.

[12] C. S. Sharp, O. Shakernia, S. S. Sastry, A vision system for landing an unmanned aerial vehicle, ICRA'01, pp. 1720 - 1727, Vol.2, Seoul, Korea, May 2001.

[13] G. F. Silveira, J. R. Azinheira, P. Rives, S. S. Bueno, Line Following Visual Servoing for Aerial Robots Combined with Complementary Sensors, ICAR'03, Coimbra, Portugal, pp. 1160-1165, July 2003.

[14] H. Zhang, J. P. Ostrowski, Visual servoing with dynamics: Control of an unmanned blimp, ICRA'99, pp. 618-623, Vol.1, Detroit, USA, May 1999. 